arrhythmia of the three groups were significantly difference. In the rest state, the ratio of abnormal myocardial perfusion segments in the three groups were statistically significant difference. The ischaemic myocardial area score group A was significantly higher than group $B$ and group $C$, group $B$ and group $C$ were no significant difference. ERNA showed that LVEF in the three groups were statistically significant difference. Group A was significantly lower than group $B$, there were no significant difference between group $B$ and group $\mathrm{C}$, group $\mathrm{A}$, group $\mathrm{C}$ differences were statistically significant illustrated the LVEF of group A was significantly lower than group B and group C.

Conclusion Glibenclamide would increase myocardial ischaemic area in patients with acute myocardial infarction and type 2 diabetes mellitus. Glibenclamide increases the possibility of malignant arrhythmias in the patients with acute myocardial infarction and type 2 diabetes mellitus.

\section{e0414 LIPID LEVEL OF PATIENTS WITH ACUTE MYOCARDIAL INFARCTION UNDERGOING PRIMARY ANGIOPLASTY IS RELATED WITH PROGNOSIS}

doi:10.1136/hrt.2010.208967.414

Liu Ran, Yan Hongbing. Beijing Anzhen Hospital

Objective TO evaluate the correlation between acute lipids level and on-statins treatment lipids and 1 year major adverse cardiovascular and cerebrovascular events (MACCE), including cardiac death, ischaemic stroke, and recurrent myocardial infarction in patients with ST-elevation myocardial infarction (STEMI) undergoing primary percutaneous coronary intervention (PCI).

Methods Based on the LDL-C level within $24 \mathrm{~h}$ after admission, consecutive 624 patients with STEMI undergoing primary PCI were classified into the normal range of LDL-C $<3.37 \mathrm{mmol} \cdot 1^{-1}(\mathrm{n}=380)$, the critical range of LDL-C $3.37 \sim 4.14 \mathrm{mmol} \cdot \mathrm{l}^{-1}(\mathrm{n}=159)$ or the elevated range of LDL-C $>4.14 \mathrm{mmol} \cdot 1^{-1} \quad(n=85)$. Of these 624 patients, serum lipids levels of 335 patients after statins treatment for 4 weeks underwent follow-up in outpatient settings. Based on the results of follow-up, these 335 patients were divided into the targeted group (LDL-C $<1.81 \mathrm{mmol} \cdot \mathrm{I}^{-1}, \mathrm{n}=77$ ) or the non-targeted group (LDL-C $\geq 1.81 \mathrm{mmol} \cdot \mathrm{l}^{-1}, \mathrm{n}=258$ ). The end point was 1 year MACCE.

Results When compared between different lipid ranges (the normal, the critical and the elevated) of patients, the acute lipid level was not associated with the end points of cardiac death, ischaemic stroke, recurrent myocardial infarction, and MACCE ( $p=0.871$, $0.568,0.978,0.587$ ). After 4 weeks statins treatment, the LDL-C level achieved the goal in 77 patients $(23.0 \%)$. The primary end point occurred in $1.3 \%$ of patients treated with LDL-C $<1.81$ $\mathrm{mmol} \cdot \mathrm{1}^{-1}$ group and in $7.4 \%$ in LDL-C $\geq 1.81 \mathrm{mmol} \cdot \mathrm{1}^{-1}$ group $(p=0.034)$. Logistic regression analysis showed a significant relationship between acute triglyceride (TG) level and ischaemic stroke (OR, 1.226; 95\% CI 1.068 to 1.407; $p=0.004$ ), and between LDL-C level after stain therapy and MACCE (OR, 1.788; 95\% CI 1.091 to 5.233; $\mathrm{p}=0.039$ ). Smoking history (OR, 0.136; 95\% CI 0.016 to $1.115 ; \mathrm{p}=0.036)$ and higher SYNTAX score (OR, 1.544; 95\% CI 5.387 to $33.522 ; \mathrm{p}=0.018$ ) were predictors of 1 year MACCE in the patients with STEMI undergoing primary PCI.

Conclusions 1 year MACCE follow-up investigation in the patients with STEMI undergoing primary PCI suggested high on-treatment LDL-C level was a high risk of increased MACCE although acute lipid level was not associated with MACCE. This finding supports the concept that achieving low LDL-C levels is an important therapeutic parameter in statins-treated patients following STEMI and PCI.

\section{e0415 THE CONTRAST RESEARCH IN SAFETY AND EFFICACY BETWEEN NADROPARIN AND DALTEPARIN BEFORE ELECTIVE PERCUTANEOUS CORONARY INTERVENTION}

doi:10.1136/hrt.2010.208967.415

Liu Ruifang, Yan Hongbing. Beijing Anzhen Hospital

Background To evaluate the contrast research in safety and effective time between nadroparin and dalteparin before elective percutaneous coronary intervention (PCI) in Chinese population. Investigating the relationship among anti-Xa activities APTT and ACT, and if it help to use nadroparin and dalteparin.

Methods A total of 101 consecutive patients undergoing elective PCI were randomised to either nadroparin (50 cases) or dalteparin (51 cases) group for procedural anticoagulation. According to description, the patients in nadroparin or dalteparin group were given nadroparin $(0.01 \mathrm{ml} / \mathrm{kg})$ or dalteparin $(120 \mathrm{IU} / \mathrm{kg})$ subcutaneously more than $48 \mathrm{~h}$ twice per day before PCI. Serial plasma anti-Xa activities ACT and APTT were measured before and after nadroparin and dalteparin were injected. Then coronary angiography (CAG) and PCI was performed. Bleeding complications were classified according to Thrombolysis in Myocardial Infarction (TIMI) criteria. All patients were monitored for anticoagulation before and after $\mathrm{PCI}$ and adverse events (ie, death, myocardial infarction, demands for revascularization) 30 days after PCI.

Results 101 patients were completed. 1. The proportion, plasma anti-Xa activities of patients in nadroparin or dalteparin group above $0.5 \mathrm{IU} / \mathrm{ml}$, exceeded $92 \%$ after $4 \mathrm{~h}$ subcutaneous injection. The proportion descended to $74 \%$ and $36 \%$ in nadroparin group after $8 \mathrm{~h}$ and $12 \mathrm{~h}, 54.9 \%$ and $23.5 \%$ in dalteparin group, which descend more, but had no significantly difference compared with nadroparin group. After $24 \mathrm{~h}$ and $48 \mathrm{~h}$, the proportion of plasma anti-Xa activities of patients in both groups are above $0.5 \mathrm{IU} / \mathrm{ml}$, achieved steady state and exceeded 96.0\%. 2. The plasma anti-Xa activities correlated with APTT significantly in both groups after $4 \mathrm{~h} 24 \mathrm{~h}$ and $48 \mathrm{~h}(\mathrm{p}<0.01)$. The plasma anti-Xa activities hardly correlated with ACT, and have no difference in Statistics (nadroparin group $p=0.075$, dalteparin group $p=0.093$ ). 3 . There were no adverse events in 30 days, and no significantly difference about bleeding events (6.0\% vs $3.9 \%$, p>0.05) between the two groups.

Conclusion The subcutaneous injection of nadroparin or dalteparin for anticoagulation in patients before elective PCI is safe and effective. Anticoagulation effects can be maintained for at least $8 \mathrm{~h}$. APTT can response to the anticoagulation effects of nadroparin or dalteparin by subcutaneous injection. ACT cannot response to the effective anticoagulation effects of nadroparin or dalteparin by subcutaneous injection.

\section{e0416 EFFECT OF CARDIOVASCULAR RISK FACTORS ON SECONDARY PERCUTANEOUS CORONARY REVASCULARIZATION}

doi:10.1136/hrt.2010.208967.416

Liu Chen, Yan Hongbing. Beïing Anzhen Hospital

Objectives An increasing number of patients undergoing percutaneous coronary intervention (PCI) have experienced previous revascularization procedures. However risk factors associated with secondary percutaneous coronary revascularization and their effect sizes were lack of research. The purpose of this large scale crosssectional survey was to investigate quantitative effect of cardiovascular risk factors.

Methods Patients with coronary heart disease hospitalised in the Department of Cardiology of Beijing Anzhen Hospital whose disease was identified by angiography were consecutively enrolled 
into the present study from January 2006 to July 2009. Demographic information, concomitant diseases, peri-operative laboratory examinations, angiographic features, and surgery information of consecutive patients who underwent PCI were collected.

Results A total of 13922 patients were recorded in the database, of which $9.03 \%(1257 / 13,922)$ had previous PCI. Univariate analysis revealed that patients with prior PCI had significant higher prevalence of hypertension, diabetes mellitus and hyperlipidaemia than those without prior PCI. In addition, the percentage of patients with prior $C A B G$ was higher in the former group (6.0\% vs $1.9 \%$, $\mathrm{p}<0.001$ ). In multivariable logistic regression analyses adjusted for demographic, clinical, angiographic and procedural factors, elderly age (OR $1.01,95 \%$ CI 1.00 to 1.01), male (OR 1.74, 95\% CI 1.48 to 2.04), hypertension (OR $1.36,95 \%$ CI 1.19 to 1.54 ), diabetes mellitus (OR $1.43,95 \%$ CI 1.24 to 1.63 ), hyperlipidaemia (OR $1.30,95 \% \mathrm{CI}$ 1.06 to 1.57 ) and prior CABG (OR 3.37, 95\% CI 2.52 to 4.51) were identified as independent risk factors of secondary PCI. Additionally, history of prior $\mathrm{CABG}$ was the most important predictor of secondary PCI.

Conclusions Risk factors associated with secondary PCI include elderly age, male, hypertension, diabetes mellitus, hyperlipidaemia and prior $\mathrm{CABG}$, of which previous $\mathrm{CABG}$ was the most important.

\section{e0417 EFFECT OF SHENGMAI INJECTION ON CARDIAC FUNCTION AND INFLAMMATORY REACTION IN PATIENTS WITH ACUTE CORONARY SYNDROME}

doi:10.1136/hrt.2010.208967.417

Zhang Yachen, Chen Man-tian, Wang Fei, Rong Yezhi, Lu Baojing. Division of Cardiology, Xinhua Hospital, School of Medicine, Shanghai Jiaotong University

Objective To confirm the effect of Shengmai injection in improving cardiac function in patients with acute coronary syndrome (ACS) and to explore its influence on inflammatory reaction in patients. Methods Ninety ACS patients were randomised into two groups, the control group, treated with conventional therapy and the SMI group, treated with SMI. The patients' cardiac function was noted and the high sensitive C-reactive protein (hs-CRP) in venous blood was measured before treatment and 1 week and 2 weeks after treatment, so as to observe and compare their changes in the two groups.

Results The cardiac output, stroke volume and ejection fraction in the SMI group after 3 weeks of treatment were all higher than those in the control group $(p<0.05)$. The serum content of hsCRP was reduced in both groups, but the reduction in the SMI group was more significant than that in the control group.

Conclusion SMI could improve cardiac function and further inhibit the inflammatory reaction in patients with ACS.

\section{e0418 EFFECT OF EECP-INTEGRATED STANDARD THERAPY FOR PATIENTS WITH STABLE CORONARY ARTERY DISEASE: A PROSPECTIVE, RANDOMISED, OPEN-LABELLED AND BLIND-ENDPOINT STUDY (PROBE-EECP TRIAL)}

doi:10.1136/hrt.2010.208967.418

Du Zhimin, Wu Guifu, Xie Qiang, Zhang Yan, Yang Daya, Ling Li, Liu Donghong, Tang Lilong, Chen Guangye, Ma Hong. Division of Cardiology The First Affiliated Hospital of Sun Yat-sen University Guangzhou

Background Enhanced External Counterpulsation (EECP) has been introduced as an noninvasive, effective and safe therapy for the management of ischaemic vascular diseases. Previously, researches had been focused on the mechanisms of EECP on vascular diseases. However, clinical data in this area are still relatively in paucity.
Objective To determine whether EECP-integrated standard therapy would reduce the major adverse cardiovascular events in patients with coronary artery diseases and improve the quality of life.

Method A total of 194 patients aged from 32 to 75 years old with coronary artery diseases from Sept. 2008 to Dec. 2009 in 4 hospitals affiliated to Sun Yat-sen University were enrolled into the trial. Subjects were randomised to be allocated either to EECP plus standard therapy group or standard therapy group, and followed for 0.5 to 1.5 -year. The coronary artery disease in all patients was documented by coronary angiography, or a history of prior myocardial infarction or prior coronary revascularization. Patients in standard therapy group were treated with guideline-driven therapeutic strategy, and patients in EECP group were given $36 \mathrm{~h}$ of EECP in addition to the guideline-driven therapeutic strategy. Repeated EECP were prescribed to patients with any reoccurred ischaemic symptoms, or new ischaemia, or no symptoms in 12 months later. Follow-up visits were performed at 1, 3, 6, 12 months and each year therefore from the inclusion.

Results The primary composite endpoints of myocardial infarction, revascularisation, readmission to hospital due to stroke and ACS occurred in 6 of 104 (5.77\%) patients in EECP group compared with 9 of $91(9.89 \%)$ in standard therapy group $(p<0.05)$. There was 1 death in both groups respectively. The incidence of minor skin damage was about $8 \%$ in EECP group, causing EECP therapy in 3 patients to be prematurely terminated.

Conclusion An EECP-integrated standard therapy significantly reduced major cardiovascular events in patients with documented coronary artery disease.

\section{e0419 THE APPLYING OF ASPIRATION CATHETER IN REVASCULARISATION IN PATIENTS WITH ST-ELEVATION MYOCARDIAL INFARCTION}

doi:10.1136/hrt.2010.208967.419

Li Weiming, Xu Yawei, Wang Ke, Chen Yangqing, Wei Yidong, Li Yuanmin, Che Wenliang, Hou Lei, Lu Yunlan. 'Department of Cardiologyshanghai Tenth People's Hospital

Objectives To observe the immediate and recent treatment effects of applying the aspiration catheter in patients with ST-elevation myocardial infarction (STEMI) undergoing primary percutaneous coronary intervention (PCI).

Methods From March to June 2010, we enrolled the STEMI patients presenting with TIMI Flow Grade 0 or 1 in the infarct related artery (IRA) at baseline CAG undergoing primary PCI. The aspiration catheter (Medtronic Inc., Export?) was applied immediately to aspirate the intracoronary thrombus. Whether predilatating and/or stenting were decided by the blood flow and the condition of lesions. The patients from October 2009 to February 2010 who was diagnosed as STEMI and received non-aspiration were enrolled as control group. The immediate and recent clinical outcomes of applying the aspiration catheter were compared between the two groups.

Results There were 25 cases in Group thrombus-aspiration (2 cases of subacute stent thrombosis). After having aspirated the intracoronary thrombus using the aspiration catheter, 14 cases of STEMI recovered immediately $(9$ cases received direct stenting; 3 cases implanted stents after balloon predilatation, and had satisfied results; 2 cases of subacute stent thrombosis received the antithrombotic therapy, included intravenous infusion of Xinweining for $36 \mathrm{~h}$ in CCU immediately after recovering TIMI grade- 3 flow). 10 cases recovered TIMI 1-2, 1 cases also showed no-reflow. All of the 11 cases received the balloon predilatation and stenting, only one showed slow flow, the others recovered. There was no other severe complication during and after the operation. There was no in-stent thrombosis during 1 month follow-up, and the cardiac function improved largely. There 